\title{
Microwave-Assisted Facile and Convenient Synthesis of Imidazolines
}

\author{
I. Mohammadpoor-Baltork ${ }^{\star}$ and M. Abdollahi-Alibeik
}

\author{
Department of Chemistry, Isfahan Universitv, Isfahan 81746-73411, Iran \\ Received April 19. 2003
}

Key Words : Nitriles, Imidazolines, Microwave irradiation

The 2-substituted imidazolines are of considerable importance because their derivatives exhibit a wide variety of biological activities, including antihypercholesterolemic. ' antiinflammatory. ${ }^{2}$ antidiabetic ${ }^{3}$ and antihypertensive. ${ }^{+}$'These compounds are also used as intermediates ${ }^{5}$ and catalysts ${ }^{6}$ in synthetic chemistry.

Several methods for the synthesis of 2-imidazolines from carboxylic acids. ${ }^{7}$ esters, ${ }^{8}$ nitriles, ${ }^{9}$ orthoesters, ${ }^{10}$ hydroximoyl chlorides, ${ }^{11}$ hydroxy amides. ${ }^{12}$ and mono-, or disubstituted chlorodicyanovinyl benzene ${ }^{1.3}$ have been reported previously. However, some of these methods suffer from disadvantages such as long reaction times, low yields of the products, difficulty in preparation of starting materials and tedious workup. Therefore, there is still a scope to find potential methods for this transformation.

In recent years organic reactions assisted by microwave irradiation have gained special attention. ${ }^{14}$ ' lhe chief features of the microwave reactions are the enhanced selectivity, much improved reaction rates. Inilder reaction conditions and formation of cleaner products. In this paper we wish to report an efficient and convenient method for the synthesis of 2-imidazolines from nitriles and ethylenediamine in the presence of sulfur under microwave irradiation (Scheme 1).

Typically, benzonitrile (1a), ethylenediamine and sulfur were mixed and irradiated for $2.5 \mathrm{~min}$ in a domestic microwave oven (operating at $720 \mathrm{~W}$ ). The reaction mixture was allowed to reach to room temperature, then cold water was added and the mixture was extracted with chloroform. Removal of the solvent and recrystallization of the crude product from cyclohexane gave the corresponding 2imidazoline (2a) in $97 \%$ yield. The effect of microwave irradiation power in this reaction was also investigated. The results show that the highest yield of compound $\mathbf{2} \mathbf{a}$ is obtained at a power of $720 \mathrm{~W}$ (Table I). At this power, the temperature of the reaction vessel after the irradiation was measured to be $-130^{\circ} \mathrm{C}$. Under the same reaction conditions. a variety of nitriles are cleanly and rapidly converted to their corresponding 2-imidazolines in $96-99 \%$ yields within 1.2-

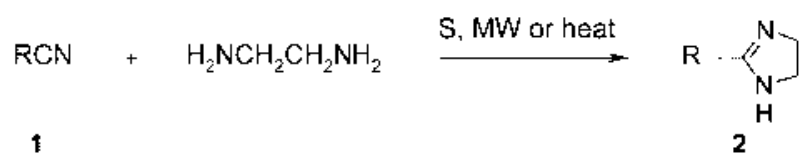

Scheme

"Corresponding author. Fax: +98-31 1-6689732; E-mail: imbaltork (a)sci.ui.ac.ir
Table 1. The cffect of microwave irradiation power on the formation $2 a^{a t}$

\begin{tabular}{lrrrr}
\hline Irradiation poticr (W) & 450 & 540 & 630 & 720 \\
\hline Yicld \% & 70 & 82 & 89 & 97 \\
\hline
\end{tabular}

"litradiation time is $2.50 \mathrm{~min}$. "lsolated yield.

I I min (Jable 2). The conventional thermal reactions were also performed for this transformation in the absence of microwave energy. The experimental results show that the yields of the products are relatively lower under this condition and the reaction times are longer, specially in the cases of $\mathbf{1 b}, \mathbf{1} \mathbf{h}$ and $\mathbf{1 j}$. In order to show the general applicability of this method, the reaction of benzonirtile was performed on a 100 minole scale. The result $(95 \%$ yield, 2.5 min) was comparable to that obtained by the small scale experiment.

In order to show the effect of sulfur, a mixture of benzonitrile (1a) and ethylenediamine was irradiated in the absence of sulfur. Under this condition, the reaction did not proceed at all and benzonitrile remained intact in the reaction mixture. The exact mechanism of the reaction is not clear at this time. However, a plausible explanation is that sulfur reacts with nitrile to produce thioamide. Thioamide reacts with ethylenediamine, which upon elimination of hydrogen sulfide and ammonia, produces 2-imidazoline. Evolution of hydrogen sulfide is a certification of the above statement.

In conclusion, a simple and efficient procedure for the synthesis of 2-imidazolines has been explored. Mild reaction conditions, absence of solvent, shorter reaction time, easy and quick isolation of the products and excellent yields are main advantages of this procedure which make this method an attractive and useful contribution to the present methodologies.

\section{Experimental Section}

General procedure for the preparation of 2-imidazolines under microwave irradiation: A mixture of nitrile (4 mmol), ethylenediamine ( $16 \mathrm{mmol}$ ) and sulfur (I mmol) was itradiated with microwave $(720 \mathrm{~W})$ for $1.2-11 \mathrm{~min}$. After completion of the reaction as indicated by TLC (eluent : EtOAc/MeOH, 4:1), the reaction mixture was cooled to room temperature, cold water was added and then extracted with chloroform. The organic layer was dried over anhydrous $\mathrm{Na}_{2} \mathrm{SO}_{4}$ and evaporated. Recrystallization of the crude 
Table 2. Preparation of 2-imidazolines from nitriles

\begin{tabular}{|c|c|c|c|c|c|c|}
\hline \multirow{2}{*}{ Eintry } & \multirow{2}{*}{ Nitrile (1) } & \multirow{2}{*}{ Imidayoline (2) } & \multicolumn{2}{|c|}{ MW } & \multicolumn{2}{|c|}{ Heat } \\
\hline & & & Timo (min) & Yíld $\%$ & Time (mits) & Yïld $\%{ }^{c t}$ \\
\hline a & $-\mathrm{CN}$ & & 2.50 & 97 & 18 & 93 \\
\hline b & 1) $-\mathrm{CN}$ & & 4.50 & 98 & 30 & 97 \\
\hline c & 1) $-\mathrm{CN}$ & & 1.30 & 97 & 7 & 96 \\
\hline d & & & 1.50 & 97 & 12 & 94 \\
\hline e & & & 2.25 & 96 & 6 & 90 \\
\hline f & $\mathrm{CN}$ & & 1.20 & 97 & 5 & 96 \\
\hline g & & & 1.20 & 96 & 3 & 91 \\
\hline h & 1) $-\mathrm{CN}$ & & 4.50 & 98 & 55 & 92 \\
\hline $\mathbf{i}$ & & & 1.30 & 99 & 15 & 97 \\
\hline j & $\mathrm{H}-\mathrm{CN}$ & & 11.00 & 97 & 150 & 97 \\
\hline
\end{tabular}

"Isolated yicld.

product from cyclohexane gave the pure product in $96-99 \%$ yields (lable 2).

General procedure for the preparation of 2-imidazolines under reflux conditions: A mixture of nitrile (4 mmol), ethylenediamine ( $16 \mathrm{mmol}$ ) and sulfur ( $1 \mathrm{mmol}$ ) was refluxed on a oil bath $\left(120^{\circ} \mathrm{C}\right)$ for $3-150 \mathrm{~min}$. The progress of the reaction was monitored by $\mathrm{TLC}$ (eluent : $\mathrm{EtOAc} / \mathrm{MeOH}$, $4:$ 1). After completion of the reaction, the mixture was cooled to room temperature and cold water was added. The reaction mixture was extracted with chloroform and the organic layer was dried over anhydrous $\mathrm{Na}_{2} \mathrm{SO}_{4}$. The solvent was evaporated and the crude product was recrystallized from cyclohexane to afford the pure product in $90-97 \%$ yields (lable 2).

Compound 2a: Mp 100-101 ${ }^{\circ} \mathrm{C}$ (Lit." $101-102$ C). 'H NMR $\left(\mathrm{ClCl}_{3}\right): \delta 3.75\left(\mathrm{~s}, 4 \mathrm{H}, 2 \mathrm{CH}_{2}\right), 4.8(\mathrm{~s}, 1 \mathrm{H}, \mathrm{NH}), 7.3-$ $7.4(\mathrm{~m}, 2 \mathrm{H}, \mathrm{ArH}), 7.7-7.8(\mathrm{~m}, 3 \mathrm{H}, \mathrm{ArH})$. IR (KBr): 3190 $(\mathrm{NH}), 1598(\mathrm{C}=\mathrm{N}) \mathrm{cm}^{-1}$. Anal. calcd. for $\mathrm{C}_{9} \mathrm{H}_{16} \mathrm{~N}_{2}: \mathrm{C}, 73.94$; H, 6.89; N. 19.16; found: C, 74.11; H, 6.84; N. 19.03 .

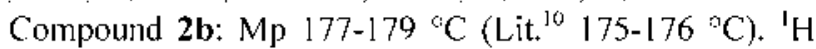
NMR (CDCl $): \delta 2.38\left(\mathrm{~s}, 3 \mathrm{H}, \mathrm{CH}_{3}\right), 3.75\left(\mathrm{~s}, 4 \mathrm{H}, 2 \mathrm{CH}_{2}\right), 4.45$ $(\mathrm{s}, 1 \mathrm{H}, \mathrm{NH}), 7.15$ (d, 2H, ArH), 7.63 (d, 2H, ArH). IR (KBr):
$3130(\mathrm{NH}), 1598(\mathrm{C}=\mathrm{N}) \mathrm{cm}^{-1}$. Anal. calcd for $\mathrm{C}_{14} \mathrm{H}_{12} \mathrm{~N}_{2}: \mathrm{C}$, 74.97; H. 7.55; N, 17.48; found: C. 74.89; H. 7.57; N. 17.53 .

Compound 2c: Mp 186-188 ${ }^{\circ} \mathrm{C}$ (Lit. ${ }^{11}$ 185-187 ${ }^{\circ} \mathrm{C}$ ). ' $\mathrm{H}$ NMR (CDCl $): \delta 3.75\left(\mathrm{~s}, 4 \mathrm{H}, 2 \mathrm{CH}_{2}\right), 4.22(\mathrm{~s}, 1 \mathrm{H}, \mathrm{NH}), 7.3$ (d, 2H, ArH), 7.93 (d. 2H, ArH). IR (KBr) $3140(\mathrm{NH}), 1590$ $(\mathrm{C}=\mathrm{N}) \mathrm{cm}^{-1}$. Anal. calcd for $\mathrm{C}_{9} \mathrm{H}_{0} \mathrm{ClN}_{2} ; \mathrm{C}, 59.84 ; \mathrm{H}, 5.02$; N. 15.51; found: C. 59.82; H, 5.06; N. 15.35.

Compound 2d: $\mathrm{Mp} 133-135^{\circ} \mathrm{C}$. ' $^{\mathrm{H}} \mathrm{NMR}\left(\mathrm{CDCl}_{3}\right): \delta 3.76$ (s. 4H, 2CH $), 4.25(\mathrm{~s}, \mathrm{lH}, \mathrm{NH}), 7.22-7.75(\mathrm{~m}, 4 \mathrm{H}, \mathrm{ArH})$. IR $(\mathrm{KBr}): 3140(\mathrm{NH}), 1595(\mathrm{C}=\mathrm{N}) \mathrm{cm}^{-1}$. Anal. calcd for $\mathrm{C}_{9} \mathrm{H}_{9} \mathrm{ClN} \mathrm{N}_{2}: \mathrm{C}, 59.84 ; \mathrm{H}, 5.02 ; \mathrm{N}: 15.51$; found: $\mathrm{C}, 59.91: \mathrm{H}$, $5.01 ; \mathrm{N}, 15.43$.

Compound 2e: Mp 134-135 ${ }^{\circ} \mathrm{C}$ (Lit. ${ }^{8} 136-137^{\circ} \mathrm{C}$ ). ${ }^{\mathrm{l}} \mathrm{H}$ NMR $\left(\mathrm{CDCl}_{3}\right): \delta 3.79\left(\mathrm{~s}, 4 \mathrm{H}_{2} 2 \mathrm{CH}_{2}\right), 4.3(\mathrm{~s}, 1 \mathrm{H}, \mathrm{NH}), 7.6 \mathrm{l}$ (d, $2 \mathrm{H}, \mathrm{ArH}), 8.65$ (d. $2 \mathrm{H}, \mathrm{ArH})$. JR (KBr): $3180(\mathrm{NH}), 1594$ $(\mathrm{C}=\mathrm{N}) \mathrm{cm}^{-1}$. Anal. calcd for $\mathrm{C}_{8} \mathrm{H}_{4} \mathrm{~N}_{3}: \mathrm{C}, 65.29 ; \mathrm{H}, 6.16 ; \mathrm{N}$. 28.55; found: C, $65.32 ; \mathrm{H}, 6.18 ; \mathrm{N}, 28.49$.

Compound 2f: $\mathrm{Mp} 111-113^{\circ} \mathrm{C}$ (Lit. ${ }^{8} 106-107{ }^{\circ} \mathrm{C}$ ). ${ }^{\mathrm{l}} \mathrm{H}$ NMR $\left(\mathrm{CDCl}_{3}\right): \delta 3.78\left(\mathrm{~s}, 4 \mathrm{H}, 2 \mathrm{CH}_{2}\right), 4.54(\mathrm{~s}, 1 \mathrm{H}, \mathrm{NH}), 7.2-$ $7.38(\mathrm{~m}, 1 \mathrm{H}, \mathrm{ArH}), 8.02-8.15(\mathrm{~m}, \mathrm{lH}, \mathrm{ArH}), 8.6-8.67(\mathrm{~m}$. 1H, ArH), $8.92(\mathrm{~s}, 1 \mathrm{H}, \mathrm{ArH})$. IR (KBr): $3150(\mathrm{NH}), 1586$ $(\mathrm{C}=\mathrm{N}) \mathrm{cm}^{-1}$. Anal. calcd for $\mathrm{C}_{8} \mathrm{H}_{9} \mathrm{~N}_{3} ; \mathrm{C}, 65.29 ; \mathrm{H}, 6.16 ; \mathrm{N}$. 
28.55; found: C. $65.29 ; \mathrm{H}, 6.20 ;$ N. 28.40

Compound 2g: Mp 101-102 ${ }^{\circ} \mathrm{C}$. ${ }^{1} \mathrm{H} N \mathrm{NM}\left(\mathrm{CDCl}_{3}\right): \delta 3.81$ (s. $\left.4 \mathrm{H}, 2 \mathrm{CH}_{2}\right) .5 .38$ (s. $1 \mathrm{H}$. NH). $7.22-7.38$ (m. 1H, $\mathrm{ArH}$ ). 7.62-7.85 (m, lH. ArH), 8.12 (d, 1H. ArH), 8.55 (d, $1 \mathrm{H}$. ArH). IR (KBr): $3240(\mathrm{NH}) .1594(\mathrm{C}=\mathrm{N}) \mathrm{cm}^{-1}$. Anal calcd for $\mathrm{C}_{8} \mathrm{H}_{9} \mathrm{~N}_{3}$ : C, 65.29 ; H. 6.16: N, 28.55; found: C. $65.25: \mathrm{H}$, 6.19: N, 28.52.

Compound 2h: Mp 139-140 ${ }^{\circ} \mathrm{C}$ (Lit. ${ }^{1]} 138-140{ }^{\circ} \mathrm{C}$ ). ${ }^{1} \mathrm{H}$ NMR ( $\left.\mathrm{CDCl}_{3}\right): \delta 3.74\left(5,4 \mathrm{H} .2 \mathrm{CH}_{2}\right) .3 .81$ (s. $\left.3 \mathrm{H}, \mathrm{CH}_{3}\right) .4 .42$ (s. 1 H. NH). 6.87 (d. $2 \mathrm{H}$. ArH), 8.7 (d. $2 \mathrm{H}$. ArH). IR (KBr): $3170(\mathrm{NH}) .1605(\mathrm{C}=\mathrm{N}) \mathrm{cm}^{-1}$. Anal. calcd for $\mathrm{C}_{10} \mathrm{H}_{12} \mathrm{~N}_{2} \mathrm{O}$ : C. 68.16: H. 6.86: N. 15.90; found: C. 68.22: H. 6.82: N. 15.75. Compound 2i: Mp 175-178 ${ }^{\circ} \mathrm{C}$ (Lit. ${ }^{8} 178-180{ }^{\circ} \mathrm{C}$ ). ${ }^{1} \mathrm{H}$ NMR $\left(\mathrm{CDCl}_{3}\right): \delta 3.75$ (s. + H. $\left.2 \mathrm{CH}_{2}\right),+25(5,1 \mathrm{H} . \mathrm{NH}), 6.9-$ 7.05 (m. 1H, ArH). 7.35-7.4 (m, 2H). IR (KBr): $3140(\mathrm{NH})$. $1597(\mathrm{C}=\mathrm{N}) \mathrm{cm}^{-1}$. Anal. calcd for $\mathrm{C}_{7} \mathrm{H}_{\S} \mathrm{N}_{2} \mathrm{~S}:$ C. $55.23 ; \mathrm{H}$. 5.30: N, 18.40; found: C. 55.12; H. 5.33: N, 18.45 .

Compound $2 \mathrm{j}$ : Mp $156-157^{\circ} \mathrm{C}$. ${ }^{1} \mathrm{H}$ NMR $\left(\mathrm{CDCl}_{3}\right): \delta 3$ (s. $1 \mathrm{H}, \mathrm{NH}) .3 .59$ (s. $\left.+\mathrm{H} .2 \mathrm{CH}_{2}\right), 5.05(\mathrm{~s}, 1 \mathrm{H}, \mathrm{CH}) .7 .25-7.3(\mathrm{~m}$. 10H. ArH). IR (KBr): $3180(\mathrm{NH}), 1594(\mathrm{C}=\mathrm{N}) \mathrm{cm}^{-1}$. Anal. calcd for $\mathrm{C}_{16} \mathrm{H}_{10} \mathrm{~N}_{2}$ : C. 81.32; H, 6.82; N. 11.85; found: C. $81.40 ; \mathrm{H}, 6.77 ; \mathrm{N} .11 .81$.

Acknowledgement. We are thankful to the Isfahan University Research Council for partial support of this work.

\section{References}

1. Li. H.-Y: Drummond, S.: DeLucea, I.: Boswell. G. A. Tetrahedron
1996.52. 11153 .

2. Ueno. M.: Imaizumi. K.: Sugita. T.: Takata. I.: Takeshita. M. $h$ ht. J. Inmunopharmac. 1995, 17, 597.

3. Rondu. F: Le Bihan. G.: Wang. X.: Lamouri. A.: Touboul, E.: Dive. G.: Bellahsene, T.: Pfeiffer. B.: Renard, P.: GuardiolaLemaitre, B.: Manechez, D.: Penicaud, L.: Ktorza, A.: Godfroid J-I. J. Med Chent 1997, 40.3793

4. Bousquet. P.: Feldman1. T. Dntgs 1999. 58. 799

5. (a) Jones. R. C. F.: Nichols. J. R. Tetrahedron Lett 1990. 31.1771 (b) Havashi. T.: Kishi. E.; Soloshonok, V. A.: Uozumi. Y Tetrahedron Lett 1996, 37, 4969. (c) Jung, M. E.: Huang, A. Org. Lett. $\mathbf{2 0 0 0}, 2,2659$

6. (a) Corey. E. T.: Grogan. M. T. Org. Lett. 1999. 1. 157. (b) Isobe. T.: Fukida. K.: Araki. Y: Ishikawa. I. Chent Conmm 2001. 243.

7. Vorbriiggen. H.: Krolikiewicz. K. Tetrahedron Lett. 1981, 22. 4471 .

8. Neef. G.: Eder U.; Sauer. G. J. Org Chem 1981. +6.2824.

9. (a) Korshin. E. E.: Sabirova. L. I.: Akhadullin. A. G.: Levin. Y. A. Russian Chem. Bull. 1994. +3.431. (b) Levesque. G.: Gressier. T. C.: Proust. M. Swnthesis 1981. 963.

10. Hill. A. J.: Johnston. J. V. J. Am. Chem. Soc. 1954. 76, 922.

11. Salgado-Zamora, H.; Campos, E.: Jimenez. R.: Cervantes, H. Hetercelcles 1998. 77,1043 .

12. Boland. N. A.: Casey. M.: Hynes. S. J.: Matthews. T. W.: Smyth. M. P. J. Org Chem. 2002. 67.3919.

13. Shin. G. I.: Lee. T. I.: Kimn J.-H. Bull. Korean Chem. Sox. 1996. 17. 29

14. (a) Caddick, K. Tetahedron 1995. 51. 10403. (b) Galema, S. A. Chem. Soc. Rew 1997, 26. 233. (c) Lidström, P. Tierney. J: Wathey. B.: Westmant. T. Tetrohe don 2001. 57.9225 . (d) MarreroTerrero. A. L. Revista CENTC Ciencias Oninticas 2000. 31. 41. (e) Kidwai. M.: Sapra. P.: Bhushan1. K. R.: Misra. P. Stmhesis 2001. 1509 